\title{
Efficacy of growth factors for the treatment of peri-implant diseases: a systematic review and meta-analysis
}

Khouly, Ismael ; Pardiñas-López, Simon ; Ruff, Ryan Richard ; Strauss, Franz-Josef

\begin{abstract}
OBJECTIVES The aim of this study was to conduct a systematic review and meta-analysis on the efficacy of growth factors (GF) on clinical outcomes after treatment (surgical/non-surgical) of peri-implant diseases (periimplant mucositis and peri-implantitis). MATERIALS AND METHODS A protocol was developed to answer the following focused question: Is there any difference for the use of GF for treatment of peri-implant diseases versus comparative GF treatment or without GF? Electronic database and manual searches were independently conducted to identify randomized controlled trials (RCTs). Publications were selected based on eligibility criteria and then assessed for risk-of-bias using the Cochrane Handbook. The primary outcome was probing depth (PD) and bleeding on probing (BOP) reduction along with changes in vertical defect depth (VDD). Changes in clinical attachment level, gingival recession, and plaque index, among others, were studied as secondary outcomes. Based on primary outcomes, random-effects meta-analysis was conducted. RESULTS A total of five RCTs were included. GF enhance the reduction of PD (standardized mean difference (SMD) $=-1.28$; 95\% confidence interval (CI) - 1.75, - 0.79; $\mathrm{p}=<0.0001)$ and BOP (SMD $=-1.23 ; 95 \% \mathrm{CI}-1.70,-0.76 ; \mathrm{p}=<0.0001)$ in the management of peri-implant mucositis. For the treatment of peri-implantitis, the use of GF yielded to significantly greater improvement in VDD $(\mathrm{SMD}=0.68 ; 95 \% \mathrm{CI} 0.22,1.14 ; \mathrm{p}=0.004)$; however, there were no significant differences in terms of PD (SMD $=0.08 ; 95 \% \mathrm{CI}-1.08,1.26 ; \mathrm{p}=0.887)$ and $\mathrm{BOP}(\mathrm{SMD}=0.211 ; 95 \% \mathrm{CI}-0.20,0.63 ; \mathrm{p}=0.317)$. The overall risk of bias of the included studies was low to unclear. CONCLUSION The results of the present systematic review suggest that the addition of GF might enhance the outcomes in the treatment of peri-implant mucositis. However, there is a lack of evidence for supporting additional benefit of GF managing peri-implantitis. CLINICAL RELEVANCE Within the limitations of the current systematic review and based on the meta-analyses, (1) the addition of GF for the treatment peri-implant mucositis might be associated with better outcomes in terms of PD and BOP, and (2) an additional benefit of GF for the treatment peri-implantitis could not be determined on the basis of the selected evidence.
\end{abstract}

DOI: https://doi.org/10.1007/s00784-020-03240-5

Posted at the Zurich Open Repository and Archive, University of Zurich

ZORA URL: https://doi.org/10.5167/uzh-190165

Journal Article

Accepted Version

Originally published at:

Khouly, Ismael; Pardiñas-López, Simon; Ruff, Ryan Richard; Strauss, Franz-Josef (2020). Efficacy of growth factors for the treatment of peri-implant diseases: a systematic review and meta-analysis. Clinical Oral Investigations, 24(7):2141-2161.

DOI: https://doi.org/10.1007/s00784-020-03240-5 
Article Type: Systematic Review

\section{EFFICACY OF GROWTH FACTORS FOR THE TREATMENT OF PERI-IMPLANT DISEASES. A SYSTEMATIC REVIEW AND META-ANALYSIS.}

\section{Running title: Growth factors in peri-implant diseases.}

Ismael Khouly, DDS, MS, PhD, ${ }^{1,2}$ Simon Pardiñas-López DDS ${ }^{3}$, MS, Ryan Richard Ruff ${ }^{4,5}$, $\mathrm{MPH}, \mathrm{PhD}$, Franz-Josef Strauss, DDS, $\mathrm{MS}^{6,7,8}$

${ }^{1}$ Associate Director of Periodontology \& Implant Dentistry, Bluestone Center for Clinical Research, New York University College of Dentistry, New York, USA.

${ }^{2}$ Clinical Assistant Professor, Department of Oral and Maxillofacial Surgery, New York University College of Dentistry, New York, USA.

${ }^{3}$ Visiting Scholar, Bluestone Clinic for Clinical Research, New York University College of Dentistry, New York, USA; and, Cell Therapy and Regenerative Medicine Group, Centre for Advanced Scientific Research (CICA) and Biomedical Research Institute of A Coruña (INIBIC) strategic group, Universidade da Coruña (UDC), University Hospital Complex of A Coruña (CHUAC), Galician Health Service (SERGAS); A Coruña, Spain.

${ }^{4}$ Associate Professor, Department of Epidemiology \& Health Promotion, New York University College of Dentistry, New York, USA.

${ }^{5}$ New York University College of Global Public Health, New York, USA.

${ }^{6}$ Department of Oral Biology, School of Dentistry, Medical University of Vienna, Vienna, Austria. ${ }^{7}$ Department of Conservative Dentistry, School of Dentistry, University of Chile, Santiago, Chile.

${ }^{8}$ Clinic of Reconstructive Dentistry, Center of Dental Medicine, University of Zurich, Zurich, Switzerland

Corresponding Author:

Ismael Khouly, DDS, MS, PhD, Associate Director of Periodontology \& Implant Dentistry, Bluestone Center for Clinical Research; Clinical Assistant Professor, Department of Oral and Maxillofacial Surgery, New York University College of Dentistry, 421 First Avenue - BCCR 2W, New York, NY 10010

Tel: +12129989453

Email: dr.ismaelkhouly@gmail.com 


\section{Conflict of Interest Statement}

The authors do not have any financial interests, either directly or indirectly, in the products or information listed in the article.

\section{Funding}

There was not funding that supported this work.

\section{Author Contribution Statement}

I. Khouly and Pardiñas-López contributed to review design, data acquisition, analysis and interpretation, and drafting and revision of the manuscript; F.J. Strauss contributed to data interpretation and drafting and revision of the manuscript; R.R. Ruff contributed to data analysis and interpretation, and drafting and revision of the manuscript. All authors gave final approval and agree to be accountable for all aspects of the work.

\section{Acknowledgements}

The authors are grateful to Mr. Richard McGowan (NYU Health Sciences Library Liaison and NYU College of Dentistry) for his assistance with the electronic database search process. 


\begin{abstract}
Objectives: The aim of this study was to conduct a systematic review and meta-analysis on the efficacy of growth factors (GF) on clinical outcomes after treatment (surgical/non-surgical) of peri-implant diseases (peri-implant mucositis and peri-implantitis).
\end{abstract}

Materials and methods: A protocol was developed to answer the following focused question: Is there any difference for the use of GF for treatment of peri-implant diseases versus comparative GF treatment or without GF? Electronic database and manual searches were independently conducted to identify randomized controlled trials (RCTs). Publications were selected based on eligibility criteria and then assessed for risk-of-bias using the Cochrane Handbook. The primary outcome was probing depth (PD) and bleeding on probing (BOP) reduction along with changes in marginal bone level (VDD). Changes in clinical attachment level, gingival recession, plaque index, among others were studied as secondary outcomes. Based on primary outcomes random-effects meta-analysis was conducted.

Results: A total of five RCTs were included. GF enhance the reduction of PD (standardized mean difference $(S M D)=-1.28 ; 95 \%$ confidence interval $(\mathrm{Cl}):-1.75,-0.79 ; p=<0.0001)$ and BOP (SMD $=-1.23 ; 95 \% \mathrm{Cl}:-1.70,-0.76 ; p=<0.0001$ ) in the management of peri-implant mucositis. For the treatment of peri-implantitis, the use of GF yielded to significantly greater decrease in VDD (SMD $=0.68 ; 95 \% \mathrm{Cl}: 0.22,1.14 ; p=0.004)$, however there were no significant differences in terms of PD (SMD $=0.08 ; 95 \% \mathrm{Cl}:-1.08,1.26 ; \mathrm{p}=0.887)$ and $\mathrm{BOP}$ $(S M D=0.211 ; 95 \% \mathrm{Cl}:-0.20,0.63 ; p=0.317)$. The overall risk of bias of the included studies was low to unclear.

Conclusion: The results of the present systematic review suggest that the addition of GF might enhance the outcomes in the treatment of peri-implant mucositis. However, there is a lack of evidence for supporting additional benefit of GF managing peri-implantitis.

Clinical Relevance: Within the limitations of the current systematic review and based on the meta-analyses: (1) the addition of growth factors for the treatment peri-implant mucositis might be associated with better outcomes in terms of probing depth and bleeding on probing, and (2) an additional benefit of growth factors for the treatment peri-implantitis could not be determined on the basis of the selected evidence.

Keywords: growth factors, peri-implant diseases, peri-implantitis, peri-implant mucositis. 


\section{BACKGROUND}

Long-term success using dental implants is the ultimate goal in implant dentistry, however, biological complications may occur, and peri-implant diseases are among the most frequent and challenging ones [1-3]. Peri-implant diseases are plaque-associated pathological conditions that affect the tissues surrounding dental implants. They are categorized into 2 types, peri-implant mucositis and peri-implantitis. Peri-implant mucositis is assumed to precede peri-implantitis, and it shows clinical signs of bleeding on probing and inflammation; whereas peri-implantitis is characterized by inflammation in the peri-implant mucosa and subsequent progressive loss of supporting bone, that could finally lead to implant failure [4]. The increasing placement of dental implants has led to an inevitable increase in the prevalence of peri-implant diseases. In a recent large-scale cross-sectional study, $32 \%$ of subjects had peri-implant mucositis and $45 \%$ had peri-implantitis [1]. This is of particular importance as the most reliable and predictable treatment of peri-implant diseases has not yet been described. As a consequence, their management is challenging, particularly for periimplantitis.

Both non-surgical and surgical treatments and regenerative procedures have been used to control peri-implant diseases, however with inconsistent results [2]. While non-surgical therapy has been performed for the treatment of both peri-implant mucositis and peri-implantitis, surgical therapy is often used to treat peri-implantitis. Non-surgical therapy is considered less effective than surgical approaches in peri-implantitis [5], nevertheless the mechanism by which one therapy outperforms the other has not yet been established. Hence, there is a clear demand to refine current treatment protocols to arrest and prevent the progression of these lesions. The main goal in the treatment of peri-implant diseases is the decontamination of the implant surface and resolution of the inflammatory process thereby arresting the progression of loss of supporting bone [6]. However, a decrease in bacterial load to a level that allows healing to occur is rather difficult using only mechanical methods since the results also depend on the access and the biofilm removal [7]. As a result, adjunctive treatments including growth factors have been suggested to improve clinical outcomes [8-14] (Fig. 1).

Growth factors used in regenerative dentistry to enhance clinical outcomes include enamel matrix derivative (EMD) and platelet derivative factors such as platelet-rich fibrin (PRF), plasma rich in growth factors (PRGF) among others. [15, 16]. They contain and stimulate a variety of bioactive molecules such as vascular endothelial growth factor (VEGF), plateletderived growth factor (PDGF), transforming growth factor beta (TGF- $\beta$ ) and bone morphogenic protein (BMP). The delivery of these growth factors is intended to transiently stimulate cells locally, promoting proliferation and differentiation and, consequently, 
regeneration [16-18]. For example, growth factors have been widely used to treat periodontal intrabony and suprabony defects, and to promote bone regeneration and bone augmentation [19-27]. Moreover, some studies indicate that these growth factors have antibacterial properties, $[28,29]$ which may provide an additional benefit due to the bacterial origin of periimplant diseases. Furthermore, the use of growth factors may improve implant-related outcomes such as implant stability and alveolar ridge preservation [30]. However, the additional effect of using growth factors in the treatment of peri-implant diseases still remains unclear.

Therefore, the aim of this study is to conduct a systematic review and meta-analysis of randomized controlled trials on the efficacy of growth factors for the treatment of peri-implant diseases.

\section{MATERIALS AND METHODS}

\section{Standardized Criteria and Type of Study}

This systematic review study was developed and designed according to the established criteria by the Cochrane collaboration [31] as well as the Preferred Reporting Items for Systematic Review and Meta-Analysis (PRISMA) criteria [32].

\section{Registry Protocol}

A review protocol was registered by the International prospective register of systematic reviews (PROSPERO) data base under the number CRD42018116547 (available at: http://www.crd.york.ac.uk/PROSPERO/display_record.php?ID=CRD42018116547).

\section{Criteria for Considering Studies for this Review}

The study question included the following PICO-T criteria: Population (P), Intervention (I), Comparison (C), Outcome (O), and Time (T)

\section{The PICO-T criteria were the following:}

- Population: Adult human subject, undergoing treatment for peri-implant diseases.

- Intervention: Growth factors in combination with surgical/non-surgical treatment.

- Comparison: comparative growth factor treatment OR no growth factor.

- Outcomes: inflammation resolution in terms of reduction of bleeding on probing, probing depth, and bone level (primary outcomes), and related parameters (e.g. gingival recession, plaque index, complications, etc.) (secondary outcomes). 
- Time: at least 3 months after treatment.

The PICO Question:

Is there any difference for the use of growth factors for surgical or non-surgical treatment of peri-implant diseases, in terms of changes on bleeding on probing, pocket depth and bone level, evaluated before and after treatment, versus comparative growth factor treatment or no growth factors, in human subjects?

\section{Eligibility Criteria}

Inclusion criteria:

Requirement for Inclusion in this study meet the following criteria:

1. Randomized controlled trials (RCT).

2. Human adult subjects ( $\geq 18$ years) undergoing treatments for peri-implant diseases using growth factors in at least one study group.

3. Presence of comparative growth factors treatment group or no growth factors control.

4. Clearly defined clinical and radiological parameters according to which peri-implant disease (peri-implant mucositis and peri-implantitis) diagnosis is made.

5. Specification of probing depth, bleeding on probing and/or marginal bone levels at least 3 months after treatment.

Exclusion criteria:

Requirement for exclusion in this study meet the following criteria:

1. Prospective cohort studies, non-randomized controlled clinical trials, retrospective cohort studies, case-control studies, case reports, systematic reviews, animal trials, letter to editors, in vivo and in vitro studies.

2. Less than 10 subjects per group.

\section{Type of outcome measures}

Primary Outcomes:

The primary outcomes were probing depth reduction (in $\mathrm{mm}$ ), bleeding on probing reduction (in per cent), and changes in marginal bone level (in $\mathrm{mm}$ ).

Secondary Outcomes: 
Secondary outcomes included the following:

- Changes in clinical attachment levels (in mm).

- Change in gingival recession (in $\mathrm{mm}$ )

- Changes in plaque index.

- Changes in gingival index.

- Changes in keratinized tissue.

- Post-operative infection.

- Complications (e.g. wound dehiscence).

- Patient reported outcome measures (e.g. pain, patient satisfaction and/or quality of life).

- Implant failures.

- Prosthetic failure.

- Adverse events related to the use of growth factors.

\section{Search methods for identification of studies}

\section{Electronics searches}

The literature searches strategy was performed in the PubMed, CINAHL, Embase and Dentistry \& and Oral Sciences Source (DOSS) electronic databases. Electronic databases including articles published up to December $15^{\text {th }}, 2019$.

Concepts and subject headings were combined for each of the database searches as detailed in Appendix I, with the help of a medical and dental librarian (RM). The search was not limited by any restrictions on language or date of publication but limited to "clinical trials". Primary and secondary concepts were developed and searched for all databases.

\section{Searching other resources}

Hand searching

The electronic search was complemented by manual searches of reference lists of selected articles and related review articles. In addition, hand searching of key related journals from January 2000 were performed: Clinical Implant Dentistry and Related Research, Clinical Oral Implant Research, Journal of Clinical Periodontology, Journal of Dental Research, Journal of Periodontal Research, Journal of Periodontology.

Unpublished studies 
Online databases providing information about clinical trials in progress were checked (clinicaltrials.gov, centerwatch.com/clinicaltrials, clinicalconnection.com). We also searched for unpublished studies in OpenGrey open access database.

\section{Data collection and analysis}

\section{Selection of studies}

Eligibility assessment was achieved through title and abstract search followed by full text review. Titles and abstracts for studies, found through the electronic database search as well as hand search and unpublished databases, were reviewed independently by two reviewers (SPL, IK) for inclusion conforming to the eligibility criteria.

After initial review, the full texts were read in detail also by two reviewers, to determine if articles were in accordance with all inclusion criteria. If the title and abstract did not provide sufficient information regarding the inclusion criteria, then the full-text papers were also reviewed. The exclusion criteria justification of the excluded studies was documented. Any disagreement was resolved by discussion, consensus and moderated by a third party (FJS).

\section{Data extraction and management}

Data were extracted by one review author (SPL) using a data form that was specifically designed for the present meta-analysis and modified for a second review process as required for presentation of additional general characteristics and primary and secondary outcomes. All data and data forms were reviewed by a second review author (IK). Consensus meetings for the selection of each paper, data collection and examination of the data entry were hold until completion of this review. The reviewers worked together to consolidate the data analysis of this review. Appendix II delineates the study characteristics recorded in the data extraction form. Corresponding authors of the included studies were contacted via email for clarification of any missing information and/or clarification of methodology and results.

\section{Assessment of risks of bias in included studies}

The methodological quality of all included randomized clinical trials was assessed according to the Cochrane Handbook for Systematic Reviews of Interventions, Chapter 8 [31]. Corresponding authors of the included studies were contacted via email for detailed information on study methodology, when key domains were assessed as unclear risk of bias by the two reviewers. Any disagreement between the reviewers was resolved after discussion, consensus, and by third party (IK). 


\section{Statistical Analysis}

For each included study, extracted data were entered into a spreadsheet and the standardized mean differences (SMD) and errors were calculated (Hedge's $g$ for bias-corrected SMDs). Means and standard deviations for studies reporting medians, minimums, and maximums and those reporting medians and interquartile ranges were computed using the method of Wan et al [33] and Hozo et al [34].

Estimates for bleeding on probing and probing depth were calculated by pooling study-specific estimates using random effects meta-analyses to account for between study heterogeneity. Analysis used the inverse variance method and $\mathrm{tau}^{2}$ estimates for the variance of the distribution of true effect sizes were estimated using the DerSimonian-Laird estimator. Vertical defect depth showed no evidence of heterogeneity and was analyzed using fixed effects metaanalysis. Subgroup analyses by condition (peri-implantitis or peri-implant mucositis) were analyzed using mixed effects models with random effects within subgroups and fixed effects between. When included studies reported multiple endpoints, the last endpoint was used. Between-study heterogeneity was assessed using the $Q$ statistic and $I^{2}$, reflecting the total proportion of variability in estimates attributed to heterogeneity. Significant $Q$ statistics or an $1^{2}$ value greater than $75 \%$ was considered to be evidence of heterogeneity. For each metaanalysis, forest plots were generated.

Statistical analyses and graphics were conducted in R v3.5.2. Statistical tests were two-sided, and significance was based off a threshold of $p<0.05$.

\section{Results}

An electronic search was completed in the following databases: Pubmed, CINAHL, Embase and Dentistry and Oral Science Sources (DOSS). The electronic search yielded a total of 4,193 results and when duplicates were removed the total results were 2,284 articles. The hand search yield to one additional relevant article. Two reviewers (SPL, IK) independently screened 2,285 abstracts, ultimately excluding 2,269 articles. Sixteen full text articles were reviewed. Eleven of the sixteen articles were excluded as they did not meet eligibility criteria (appendix table 1)[35-45]. The remaining five studies were included in the present systematic review group [14, 46-49] (Appendix Figure 1).

\section{Description of included studies}

\section{General characteristics of included studies}


A total of five trials met the aforementioned eligibility criteria and were included in this systematic review. The included studies consisted in 201 patients receiving treatment in 220 implants. The included trials were carried out in academic clinical settings across the world. Average age and gender distributions were comparable across the studies. Study characteristics including study design, treatment groups, study site and surgical protocol are shown in table 1.

Three trials examined the effect of using GF during surgical therapy of peri-implantitis, while two trials examined the effect of GF during non-surgical therapy of peri-implant mucositis. Autogenous Growth Factors were used in two trials for the treatment of peri-implantitis, and EMD in three trials (one for treatment of peri-implantitis and two for peri-implant mucositis).

Surgical treatment of peri-implantitis consisted mainly in mechanical debridement with titanium instruments, while implant surface decontamination methods were different in these three trials. Pre-treatment was reported in four trials. Definitions of peri-implant diseases were slightly different across the trials. Isler et al., 2018 [14] and Hamzacebi et al. 2015 [49] used the same definition for peri-implantitis, while Isehed et al 2016 [48] slightly different. Faramarzi et al. 2015 and Kashefimehr et al. 2017 used the same definition for peri-implant mucositis and mild peri-implantitis $[46,47]$.

\section{Effects of interventions}

Tables 2 and 3 summarize the primary and secondary outcomes for included studies respectively.

Individual study outcomes

\section{Results of primary outcomes}

\section{Peri-implant mucositis}

EMD in non-surgical therapy

Two studies examined the addition of EMD for the non-surgical treatment of peri-implant mucositis. One trial reported statistically significant improvements in terms of PD and BOP when EMD was added to non-surgical treatment at 3 months as compared to the control group [46]. However, the therapy had a limited effect on both groups. Similarly, in another trial the addition of EMD significantly improved the results of the non-surgical therapy in terms of PD and BOP at 3 months follow-up [47]. 


\section{Peri-implantitis}

EMD in surgical therapy

In one study the addition of EMD to open flap debridement failed to show additional benefits in terms of PD, BOP and marginal bone level at 12-month follow-up [48]. The same cohort was followed up for 3 and 5 years but still without significant differences for the aforementioned clinical parameters [50].

Concentrated growth factor (CGF) and platelet-rich fibrin (PRF) in surgical therapy

One RCT examined the clinical benefits of GF membranes versus a regular collagen membrane in peri-implantitis defects [14]. The use of growth factor membranes in conjunction with DBBM rendered significantly inferior results as compared to DBBM plus collagen membrane in terms of PD and vertical defect depth (VDD) but no differences in BOP were found at 12 months. In another study, the addition of PRF to open flap debridement rendered more favorable results compared to open flap debridement alone in terms of PD [49].

\section{Results of secondary outcomes}

\section{Peri-implant mucositis}

EMD in non-surgical therapy

One trial exhibited statistically significant improvements in the levels of local plaque index but not in the whole-plaque index when EMD added to non-surgical treatment at 3 months [46].

\section{Peri-implantitis}

\section{EMD in surgical therapy}

The addition of EMD to open flap debridement did not significantly improve the results in terms of plaque levels, presence of pus (infection) and implant failures as compared to the control group at 1-, 3- and 5-year follow-ups [48, 50].

Concentrated growth factor (CGF) and platelet-rich fibrin (PRF) in surgical therapy

The use of growth factor membranes in conjunction with DBBM rendered significantly inferior results as compared to DBBM plus collagen membrane in terms of CAL but no significant differences were found in terms of plaque index, gingival index and mucosal recession at 12 months follow-up [14]. The addition of PRF to open flap debridement rendered more favorable 
results compared to open flap debridement alone in terms of CAL at 3 and 6 months [49]. Furthermore, PRF significantly increased the keratinized mucosa and significantly reduced the recessions after treatment at 6 months [49].

\section{Pooled Data}

Meta-analysis was attempted for primary and all secondary study outcomes. Results from the meta-analysis revealed that GF enhance the reduction of PD (SMD $=-1.28 ; 95 \% \mathrm{Cl}$ : $-1.75,-$ $0.8 ; \mathrm{p}<0.0001$ ) (Fig. 2) and BOP (SMD = -1.23; 95\% Cl: -1.71, -0.76; p < 0.0001) (Fig. 3) in the treatment peri-implant mucositis. For the treatment of peri-implantitis, the meta-analysis reported a significantly greater increase in bone levels $(\mathrm{SMD}=0.68 ; 95 \% \mathrm{Cl}: 0.22,1.14 ; \mathrm{p}=$ 0.004 ) (Fig. 4) by the use of GF, however there were no significant differences in terms of PD $(\mathrm{SMD}=0.08 ; 95 \% \mathrm{Cl}:-1.09,1.26 ; \mathrm{p}=0.887)$ (Fig.5) and BOP (SMD $=0.21 ; 95 \% \mathrm{Cl}:-0.20$, $0.63 ; p=0.317$ ) (Fig. 6). There were no significant differences in terms of PD (SMD = -0.46; $95 \% \mathrm{Cl}:-1.41,0.49 ; \mathrm{p}=0.34$ ) (Fig.7) and BOP (SMD = -0.49; 95\% Cl: $-1.31,0.32 ; \mathrm{p}=0.23$ ) (Fig. 8) combining treatment of peri-implant mucositis and peri-implantitis. Analysis of pooled data could not be conducted for any secondary outcomes as, to our knowledge, no other trials examining comparative study groups and reporting on comparable outcomes have been published.

\section{Risk of bias of included studies}

All included studies were designed as RCTs. The overall risk of bias of the included studies was low to unclear (Fig. 9a). The risk of bias assessed for seven key domains was unclear from one to three key domains in three trials (Fig. 9b) [46, 47, 49]. Two studies showed low risk of bias in all domains $[14,48]$.

\section{Discussion}

\section{Summary of key findings}

This systematic review was focused on evaluating the therapeutic value of growth factors in conjunction with non-surgical and surgical modalities for the treatment for peri-implant related diseases. Regarding peri-implant mucositis, our meta-analysis showed that the addition of EMD rendered superior results relative to the control groups in terms of BOP (SMD $=-1.23$; $95 \% \mathrm{Cl}:-1.71,-0.76 ; \mathrm{p}<0.0001)$. Furthermore, EMD reduced the PD to a higher extent in peri-implant mucositis (SMD $=-1.28 ; 95 \% \mathrm{Cl}:-1.76,-0.8 ; \mathrm{p}<0.0001)$.

The present meta-analysis, however, indicated that growth factors failed to produce an additional benefit in terms of PD (SMD $=0.08 ; 95 \% \mathrm{Cl}:-1.09,1.26 ; p=0.887)$ and BOP (SMD 
$=0.21 ; 95 \% \mathrm{Cl}:-0.20,0.63 ; \mathrm{p}=0.317)$ in the treatment of peri-implantitis. Interestingly, the current analysis also found that the addition of EMD improved the outcomes in terms of VDD in subjects affected by peri-implantitis (SMD $=0.68 ; 95 \% \mathrm{Cl}: 0.22,1.14 ; \mathrm{p}=0.0040)$.

\section{Quality of the evidence}

The overall risk of bias of the included studies was low to unclear. Two studies showed low risk of bias in all domains whereas other two studies only the allocation concealment was unclear. Only one study did not provide a detailed report of three key domains. Altogether, these observations suggest a plausible bias that raises some doubt about the results. Therefore, the information presented in this systematic review should be interpreted with caution.

\section{Potential bias in the review process}

The case definition for peri-implant diseases is highly variable for peri-implant mucositis or peri-implantitis and, until recently, there was no single uniform definition of peri-implantitis. The recent world workshop of periodontal and peri-implant diseases and conditions has provided a definition for peri-implant mucositis and peri-implantitis [51]. However, none of the included studies used the proposed definition. Concerning peri-implantitis, two $[14,49]$ out of three studies defined it as follows: $P D \geq 5 \mathrm{~mm}+\geq 2 \mathrm{~mm}$ of peri-implant bone loss combined with BOP. Conversely, Isehed et al. [48] defined peri-implantitis as if the patient had at least one implant with $\mathrm{PD} \geq 5 \mathrm{~mm}+\geq 3 \mathrm{~mm}$ of peri-implant bone loss. This difference in case definition may have an impact on the outcomes and in the extrapolation of the data, especially when the definition of peri-implantitis has been questioned [52].

According to some authors [52], peri-implantitis should be redefined, implying a change of paradigm. As such, peri-implantitis would be associated to a foreign body reaction that is not counterbalanced by the host-immune response [53]. Consequently, mucositis and bone loss around implants may represent normal conditions and not necessarily a state of diseases [52]. Hence, the question arises whether the patients included in the present review really suffered from a disease and not just an immune response.

\section{Agreements and disagreements with other previously published articles}

To date, there are few studies evaluating the effect of GF in the treatment of peri-implant diseases. Consequently, the comparison with other reviews in terms of disease resolution outcomes is limited. 
In general, the additional effect of GF in terms of BOP and PD reduction are considerably better as compared to previous systematic reviews for peri-implant mucositis treatment. Outcomes from one systematic review showed that the adjunctive or alternative use of glycine powder air polishing failed to provide an additional improvement in BOP in the treatment of PIM [54]. Similarly, findings from another systematic review indicated that adjunctive antiseptics/antibiotics (local and systemic) does not improve BOP and PD significantly [55], and outcomes of a more recent systematic review suggest that non-surgical treatment is effective for peri-implant mucositis independent of adjunctive therapy (e.g., local antiseptics, systemic antibiotics, air abrasive device) [56]. This differs from the results obtained by the current review since reduction of BOP and PD in peri-implant mucositis was not achieved without the addition of GF. Indeed, a 3-month RCT indicates that non-surgical debridement yields a complete resolution of BOP in only $38 \%$ of the implants diagnosed with peri-implant mucositis-[57].

With respect to peri-implantitis, the current data synthesis revealed that the addition of GF failed to provide an additional effect in BOP and PD. This lack of difference might be explained due to methodological discrepancies between the selected studies. For example, Isler et al. $[48,50]$ used GF in conjunction with bone grafts, while the other studies [48-50] did not include bone grafting procedures. This may have influenced the potential benefit of GF. Furthermore, one of the included studies was underpowered for the chosen design, thereby impeding the detection of an effect $[48,50]$. However, the results of the present meta-analysis are in line with recent systematic reviews focused on different therapies for the management of periimplantitis. For instance, findings from a systematic review revealed that there was insufficient evidence for supporting additional benefit of reconstructive therapy to other treatment modalities of peri-implantitis management [58]. Results from another systematic review, which evaluated adjunctive measures to conventional treatment of peri-implantitis, failed to show any significant differences in terms of BOP and PD [55]. These observations were further confirmed in a more recent systematic review [59] where the surgical treatment in periimplantitis did not provide significant differences in terms of PD or BOP [59].

Interestingly, the present meta-analysis found that the addition of EMD improved the outcomes in terms of VDD in subjects affected by peri-implantitis $(S M D=0.68 ; 95 \% \mathrm{Cl}: 0.22,1.14 ; p=$ 0.0040). These positive observations match those reported in a case series study where the combination of EMD and PDGF in combination with bone grafts increased bone fill [60]. Our results are also consistent with a recent systematic review where a surgical procedure with implantoplasty improved radiographic outcomes [61]. However, unlike our meta-analysis, the authors also reported a significant difference in BOP and PD. Those significant differences 
could be attributed to the implantoplasty itself and not to the use GF. In fact, the selected studies in the present review did not include implantoplasty or any modification of the titanium surface.

\section{Limitations and confounding variables}

The present review has some limitations. First, there was a small number of RCTs using growth factors in the treatment of peri-implant diseases. Moreover, most of the studies included small sample sizes and were underpowered, thereby reducing the possibility of detecting clinically relevant effects. Second, the clinical outcomes following surgical treatment of peri-implantitis may depend on the defect morphology. The included studies provided limited information on the morphology of treated defects. Third, there were inconsistencies in methodology, specifically various treatment modalities with different implant systems along with different follow-up periods. Hence, there is a clear demand for randomized controlled studies with proper designs and larger sample sizes, comparing the surgical or non-surgical treatment of peri-implant diseases with or without growth factors, to provide stronger evidence about their possible additional benefit.

The selected studies included different implants systems, with different surfaces either cemented or screw-retained, thereby affecting not only the quality of the decontamination but also the flap design. Concerning peri-implantitis, there is some evidence indicating that nonmodified surfaces yield better results following surgical treatment [62, 63] likely due to a different bacterial adhesion [64]. For example, the use of antibiotics may have a stronger effect in non-modified surface compared to modified ones [63]. To overcome this issue, the removal of the modified-surface has been recommended, a procedure termed implantoplasty [65]. None of the selected studies included implantoplasty, although multiple surfaces were included and in two out of five studies antibiotics were given. This may stand as one of the reasons why we failed to find significant difference in terms of BOP and PD in peri-implantitis. It should be mentioned, however, that the use of $\mathrm{PD}$ as a parameter for disease resolution has been questioned [66, 67]. Following treatment of peri-implantitis the resolution of the disease may arrest progressive bone loss, reduce BOP and/or suppuration but maintaining deep PD due to previous marginal loss [68]. In this sense, a precise physiological PD at implant sites is difficult to establish [66].

Prosthetic design also plays a pivotal role as the prosthetic design affects the oral hygiene [6], however, two out of the six included studies did not provide enough information about the restoration which may have influenced the outcomes. 
The centrifugation force and time lead to different PRF-based matrices with biological differences [69]. Consequently, the use of different protocols inevitably complicates the comparison between the included studies. In addition, the number of clots or membranes utilized during the procedure might influence the clinical outcomes [30, 70]. Isler et al. [14] reported the use of 2 membranes, Hamzacebi et al. [49] did not report it.

The position of the implants seems to be a predisposing factor to develop peri-implant mucositis and peri-implantitis due to the difficulty for oral hygiene [66], nevertheless the type of restoration that was performed in each study is unclear. Another controversial aspect is the amount of keratinized tissue which may affect the development and the long-term outcomes [71-73]. Not every study reported on the amount of keratinized tissue. Recent studies, however, have failed to show any significant advantage of the amount of keratinized tissue-

\section{Conclusions}

\section{Implications for Clinical Practice}

Within the limitations of the current systematic review and based on the meta-analyses: (1) the addition of growth factors for the treatment peri-implant mucositis might be associated with better outcomes in terms of probing depth and bleeding on probing, and (2) an additional benefit of growth factors for the treatment peri-implantitis could not be determined on the basis of the selected evidence. Hence, no definite conclusion may be drawn on the use of growth factors for the treatment of peri-implant diseases.

\section{Future research}

There is a need for further properly designed and conducted randomized clinical trials aimed at evaluating the effect of different growth factors for the treatment of peri-implant diseases. These clinical trials should incorporate the same definition of peri-implant diseases with reproductible outcome assessment methods. Additionally, these studies should be conducted to assess: 1) the efficacy of growth factors compared to another growth factor or no growth factors (or placebo), 2) the efficacy of combining various growth factors during the treatment of peri-implant diseases, and 3) the effect of local and systemic factors on outcomes of interest such as implant surface, anatomical morphology, soft tissue characteristics, etc.

\section{Clinical Relevance}

Scientific rationale for study: It is not clear if the use of growth factors for surgical or nonsurgical treatment of peri-implant diseases are more effective than comparative growth factors treatment or without growth factors. 
Principal findings: addition of growth factors might enhance the outcomes in the treatment of peri-implant mucositis, although its benefit-cost ratio must be further investigated. Practical implications: based on current evidence, no definite conclusion may be drawn on the use of growth factors for the treatment of peri-implant diseases. Hence, there is a need for further clinical trials aimed at evaluating the effect of different growth factors for the treatment of periimplant diseases. 


\section{COMPLIANCE WITH ETHICAL STANDARDS}

CONFLICT OF INTEREST: The authors of this paper declare that they have no conflict of interest.

FUNDING: There was not funding that supported this work.

ETHICAL APPROVAL: this article does not contain any studies with human participants or animals performed by any of the authors, ethical approval is not required.

INFORMED CONSENT: for this type of study, formal consent is not required. 


\section{REFERENCES}

1. Derks, J. and C. Tomasi, Peri-implant health and disease. A systematic review of current epidemiology. J Clin Periodontol, 2015. 42 Suppl 16: p. S158-71.

2. Figuero, E., et al., Management of peri-implant mucositis and peri-implantitis. Periodontol 2000, 2014. 66(1): p. 255-73.

3. Jepsen, S., et al., Primary prevention of peri-implantitis: managing peri-implant mucositis. J Clin Periodontol, 2015. 42 Suppl 16: p. S152-7.

4. Berglundh, T., et al., Peri-implant diseases and conditions: Consensus report of workgroup 4 of the 2017 World Workshop on the Classification of Periodontal and Peri-Implant Diseases and Conditions. J Periodontol, 2018. 89 Suppl 1: p. S313-S318.

5. Berglundh, T., et al., Peri-implantitis and its prevention. Clin Oral Implants Res, 2019. 30(2): p. 150-155.

6. Serino, G. and C. Strom, Peri-implantitis in partially edentulous patients: association with inadequate plaque control. Clin Oral Implants Res, 2009. 20(2): p. 169-74.

7. Renvert, S., A.M. Roos-Jansaker, and N. Claffey, Non-surgical treatment of peri-implant mucositis and peri-implantitis: a literature review. J Clin Periodontol, 2008. 35(8 Suppl): p. 305-15.

8. Renvert, S., et al., Mechanical and repeated antimicrobial therapy using a local drug delivery system in the treatment of peri-implantitis: a randomized clinical trial. J Periodontol, 2008. 79(5): p. 836-44.

9. Schar, D., et al., Anti-infective therapy of peri-implantitis with adjunctive local drug delivery or photodynamic therapy: six-month outcomes of a prospective randomized clinical trial. Clin Oral Implants Res, 2013. 24(1): p. 104-10.

10. Buchter, A., et al., Sustained release of doxycycline for the treatment of peri-implantitis: randomised controlled trial. Br J Oral Maxillofac Surg, 2004. 42(5): p. 439-44.

11. Hallstrom, H., et al., Open flap debridement of peri-implantitis with or without adjunctive systemic antibiotics: A randomized clinical trial. J Clin Periodontol, 2017. 44(12): p. 12851293.

12. Schwarz, F., et al., Clinical evaluation of an Er:YAG laser for nonsurgical treatment of periimplantitis: a pilot study. Clin Oral Implants Res, 2005. 16(1): p. 44-52.

13. Wohlfahrt, J.C., et al., Porous titanium granules in the surgical treatment of peri-implant osseous defects: a randomized clinical trial. Int J Oral Maxillofac Implants, 2012. 27(2): p. 401-10.

14. Isler, S.C., et al., Regenerative surgical treatment of peri-implantitis using either a collagen membrane or concentrated growth factor: A 12-month randomized clinical trial. Clin Implant Dent Relat Res, 2018. 20(5): p. 703-712.

15. Larsson, L., et al., Regenerative Medicine for Periodontal and Peri-implant Diseases. J Dent Res, 2016. 95(3): p. 255-66.

16. Miron, R.J., et al., Use of platelet-rich fibrin in regenerative dentistry: a systematic review. Clin Oral Investig, 2017. 21(6): p. 1913-1927.

17. Jiang, J., et al., Effects of enamel matrix derivative on gene expression of primary osteoblasts. Oral Surg Oral Med Oral Pathol Oral Radiol Endod, 2001. 91(1): p. 95-100.

18. Strauss, F.J., et al., Effect of platelet-rich fibrin on cell proliferation, migration, differentiation, inflammation, and osteoclastogenesis: a systematic review of in vitro studies. Clin Oral Investig, 2019.

19. Heijl, L., et al., Enamel matrix derivative (EMDOGAIN) in the treatment of intrabony periodontal defects. J Clin Periodontol, 1997. 24(9 Pt 2): p. 705-14.

20. Di Tullio, M., et al., Treatment of supra-alveolar-type defects by a simplified papilla preservation technique for access flap surgery with or without enamel matrix proteins. J Periodontol, 2013. 84(8): p. 1100-10. 
21. Miron, R.J., et al., Twenty years of enamel matrix derivative: the past, the present and the future. J Clin Periodontol, 2016. 43(8): p. 668-83.

22. Sculean, A., et al., Biomaterials for promoting periodontal regeneration in human intrabony defects: a systematic review. Periodontol 2000, 2015. 68(1): p. 182-216.

23. Del Fabbro, M., et al., Autologous platelet concentrate for post-extraction socket healing: a systematic review. Eur J Oral Implantol, 2014. 7(4): p. 333-44.

24. Del Fabbro, M., G. Gallesio, and M. Mozzati, Autologous platelet concentrates for bisphosphonate-related osteonecrosis of the jaw treatment and prevention. A systematic review of the literature. Eur J Cancer, 2015. 51(1): p. 62-74.

25. Khouly, I., et al., Long-Term Implant Survival After 100 Maxillary Sinus Augmentations Using Plasma Rich in Growth Factors. Implant Dent, 2017. 26(2): p. 199-208.

26. Hammarstrom, L., L. Heijl, and S. Gestrelius, Periodontal regeneration in a buccal dehiscence model in monkeys after application of enamel matrix proteins. J Clin Periodontol, 1997. 24(9 Pt 2): p. 669-77.

27. Pardinas Lopez, S., O. locca, and I. Khouly, Three-dimensional bone evaluation after surgical treatment with plasma rich in growth factors of Medication Related Osteonecrosis of the Jaw (MRONJ): A report of 3 cases. Bone Rep, 2019. 10: p. 100208.

28. Walter, C., et al., Moderate effect of enamel matrix derivative (Emdogain Gel) on Porphyromonas gingivalis growth in vitro. Arch Oral Biol, 2006. 51(3): p. 171-6.

29. Anitua, E., et al., Antibacterial effect of plasma rich in growth factors (PRGF(R)-Endoret(R)) against Staphylococcus aureus and Staphylococcus epidermidis strains. Clin Exp Dermatol, 2012. 37(6): p. 652-7.

30. Strauss, F.J., A. Stahli, and R. Gruber, The use of platelet-rich fibrin to enhance the outcomes of implant therapy: A systematic review. Clin Oral Implants Res, 2018. 29 Suppl 18: p. 6-19.

31. Higgins, J.P., Cochrane handbook for systematic reviews of interventions. Version 5.1.0 [updated March 2011]. The Cochrane Collaboration. www.cochrane-handbook.org, 2011.

32. Moher, D., et al., Preferred reporting items for systematic reviews and meta-analyses: the PRISMA statement. PLoS Med, 2009. 6(7): p. e1000097.

33. Wan, X., et al., Estimating the sample mean and standard deviation from the sample size, median, range and/or interquartile range. BMC Med Res Methodol, 2014. 14: p. 135.

34. Hozo, S.P., B. Djulbegovic, and I. Hozo, Estimating the mean and variance from the median, range, and the size of a sample. BMC Medical Research Methodology, 2005. 5(1): p. 13.

35. Khan, Z.A., et al., Evaluation of peri-implant tissues around nanopore surface implants with or without platelet rich fibrin: a clinico-radiographic study. Biomed Mater, 2018. 13(2): p. 025002.

36. Diana, C., et al., Does platelet-rich fibrin have a role in osseointegration of immediate implants? A randomized, single-blind, controlled clinical trial. Int J Oral Maxillofac Surg, 2018. 47(9): p. 1178-1188.

37. Shah, R., et al., A novel approach to treat peri implantitis with the help of PRF. Pan Afr Med J, 2017. 27: p. 256.

38. Wang, Y., et al., Enamel matrix derivative improves gingival fibroblast cell behavior cultured on titanium surfaces. Clin Oral Investig, 2016. 20(4): p. 685-95.

39. Froum, S.J., S.H. Froum, and P.S. Rosen, A Regenerative Approach to the Successful Treatment of Peri-implantitis: A Consecutive Series of 170 Implants in 100 Patients with 2- to 10-Year Follow-up. Int J Periodontics Restorative Dent, 2015. 35(6): p. 857-63.

40. Qu, Z., et al., Effect of enamel matrix derivative on proliferation and differentiation of osteoblast cells grown on the titanium implant surface. Oral Surg Oral Med Oral Pathol Oral Radiol Endod, 2011. 111(4): p. 517-22.

41. Sculean, A., Windsich, P., Auschill, M., Döri, F., Treatment of Peri-Implantitis with EDTADecontamination and Application of anEnamel Matrix Protein Derivative -a Report of 3 Cases. Periodontal Practice Today, 2004. 1(3): p. 237-245. 
42. Simion, M., et al., Evaluation of a resorbable collagen matrix infused with rhPDGF-BB in periimplant soft tissue augmentation: a preliminary report with 3.5 years of observation. Int J Periodontics Restorative Dent, 2012. 32(3): p. 273-82.

43. Kaigler, D., et al., Platelet-derived growth factor applications in periodontal and peri-implant bone regeneration. Expert Opin Biol Ther, 2011. 11(3): p. 375-85.

44. Meraw, S.J., et al., Treatment of peri-implant defects with combination growth factor cement. J Periodontol, 2000. 71(1): p. 8-13.

45. Salcetti, J.M., et al., The clinical, microbial, and host response characteristics of the failing implant. Int J Oral Maxillofac Implants, 1997. 12(1): p. 32-42.

46. Kashefimehr, A., et al., Effects of enamel matrix derivative on non-surgical management of peri-implant mucositis: a double-blind randomized clinical trial. Clin Oral Investig, 2017. 21(7): p. 2379-2388.

47. Faramarzi, M., et al., Microbiological and clinical effects of enamel matrix derivative and sustained-release micro-spherical minocycline application as an adjunct to non-surgical therapy in peri-implant mucosal inflammation. J Korean Assoc Oral Maxillofac Surg, 2015. 41(4): p. 181-9.

48. Isehed, C., et al., Effectiveness of enamel matrix derivative on the clinical and microbiological outcomes following surgical regenerative treatment of peri-implantitis. A randomized controlled trial. J Clin Periodontol, 2016. 43(10): p. 863-73.

49. Hamzacebi, B., B. Oduncuoglu, and E.E. Alaaddinoglu, Treatment of Peri-implant Bone Defects with Platelet-Rich Fibrin. Int J Periodontics Restorative Dent, 2015. 35(3): p. 415-22.

50. Isehed, $\mathrm{C}$., et al., Surgical treatment of peri-implantitis using enamel matrix derivative, an RCT: 3- and 5-year follow-up. J Clin Periodontol, 2018. 45(6): p. 744-753.

51. Renvert, S., et al., Peri-implant health, peri-implant mucositis, and peri-implantitis: Case definitions and diagnostic considerations. J Periodontol, 2018. 89 Suppl 1: p. S304-S312.

52. Albrektsson, T., et al., Initial and long-term crestal bone responses to modern dental implants. Periodontol 2000, 2017. 73(1): p. 41-50.

53. Albrektsson, T., D. Buser, and L. Sennerby, Crestal bone loss and oral implants. Clin Implant Dent Relat Res, 2012. 14(6): p. 783-91.

54. Schwarz, F., K. Becker, and S. Renvert, Efficacy of air polishing for the non-surgical treatment of peri-implant diseases: a systematic review. J Clin Periodontol, 2015. 42(10): p. 951-9.

55. Schwarz, F., A. Schmucker, and J. Becker, Efficacy of alternative or adjunctive measures to conventional treatment of peri-implant mucositis and peri-implantitis: a systematic review and meta-analysis. Int J Implant Dent, 2015. 1(1): p. 22.

56. Suarez-Lopez Del Amo, F., S.H. Yu, and H.L. Wang, Non-Surgical Therapy for Peri-Implant Diseases: a Systematic Review. J Oral Maxillofac Res, 2016. 7(3): p. e13.

57. Heitz-Mayfield, L.J., et al., Anti-infective treatment of peri-implant mucositis: a randomised controlled clinical trial. Clin Oral Implants Res, 2011. 22(3): p. 237-41.

58. Khoshkam, V., et al., Reconstructive procedures for treating peri-implantitis: a systematic review. J Dent Res, 2013. 92(12 Suppl): p. 131S-8S.

59. Ramanauskaite, A., et al., Surgical Non-Regenerative Treatments for Peri-Implantitis: a Systematic Review. J Oral Maxillofac Res, 2016. 7(3): p. e14.

60. Froum, S.J., S.H. Froum, and P.S. Rosen, Successful management of peri-implantitis with a regenerative approach: a consecutive series of 51 treated implants with 3- to 7.5-year followup. Int J Periodontics Restorative Dent, 2012. 32(1): p. 11-20.

61. Keeve, P.L., et al., Surgical Treatment of Periimplantitis With Non-Augmentative Techniques. Implant Dent, 2019. 28(2): p. 177-186.

62. Carcuac, O., et al., Surgical treatment of peri-implantitis: 3-year results from a randomized controlled clinical trial. J Clin Periodontol, 2017. 44(12): p. 1294-1303. 
63. Carcuac, O., et al., Adjunctive Systemic and Local Antimicrobial Therapy in the Surgical Treatment of Peri-implantitis: A Randomized Controlled Clinical Trial. J Dent Res, 2016. 95(1): p. 50-7.

64. Fickl, S., et al., Experimental Peri-Implantitis around Different Types of Implants - A Clinical and Radiographic Study in Dogs. Clin Implant Dent Relat Res, 2015. 17 Suppl 2: p. e661-9.

65. Renvert, S. and I.N. Polyzois, Clinical approaches to treat peri-implant mucositis and periimplantitis. Periodontol 2000, 2015. 68(1): p. 369-404.

66. Berglundh, T., et al., Peri-implant diseases and conditions: Consensus report of workgroup 4 of the 2017 World Workshop on the Classification of Periodontal and Peri-Implant Diseases and Conditions. J Clin Periodontol, 2018. 45 Suppl 20: p. S286-S291.

67. Doornewaard, R., et al., How do peri-implant biologic parameters correspond with implant survival and peri-implantitis? A critical review. Clin Oral Implants Res, 2018. 29 Suppl 18: p. 100-123.

68. Bianchini, M.A., et al., Two to six-year disease resolution and marginal bone stability rates of a modified resective-implantoplasty therapy in 32 peri-implantitis cases. Clin Implant Dent Relat Res, 2019.

69. Fujioka-Kobayashi, M., et al., Optimized Platelet-Rich Fibrin With the Low-Speed Concept: Growth Factor Release, Biocompatibility, and Cellular Response. J Periodontol, 2017. 88(1): p. 112-121.

70. Castro, A.B., et al., Regenerative potential of leucocyte- and platelet-rich fibrin. Part B: sinus floor elevation, alveolar ridge preservation and implant therapy. A systematic review. J Clin Periodontol, 2017. 44(2): p. 225-234.

71. Lang, N.P. and H. Loe, The relationship between the width of keratinized gingiva and gingival health. J Periodontol, 1972. 43(10): p. 623-7.

72. Monje, A. and G. Blasi, Significance of keratinized mucosa/gingiva on peri-implant and adjacent periodontal conditions in erratic maintenance compliers. J Periodontol, 2019. 90(5): p. 445-453.

73. Wennstrom, J.L., Lack of association between width of attached gingiva and development of soft tissue recession. A 5-year longitudinal study. J Clin Periodontol, 1987. 14(3): p. 181-4. 


\section{LEGENDS}

Table 1. Detailed data of included studies.

Table 2. Detailed primary outcome data of included studies.

Table 3. Detailed secondary outcome data of included studies.

Figure 1. Drawing illustrating, step by step, the development and treatment of peri-implantitis: A: Healthy implant with intact peri-implant tissues; B: Peri-implantitis showing tissue inflammation, bone loss around the implant, bacterial accumulation in the implant surface and the macrophagues and lymphocytes activity; C: Bacteria accumulation on the implant surface; D: Close-up view of the processes that occur due to peri-implantitis. This includes presence of bacteria, soft tissue inflammation, bleeding, osteclastic activity that leads to bone loss; $\mathrm{E}$ : Mechanical decontamination of the implant surface; F: Treatment of peri-implantitis; G: Healing of the peri-implant tissues after treatment; $\mathrm{H}$ : Close-up view of the processes that occur during healing of the peri-implant tissues.

Figure 2. Forest Plot for PD outcome in peri-implant mucositis.

Figure 3. Forest Plot for BOP outcome in peri-implant mucositis.

Figure 4. Forest Plot for VDD outcome in peri-implantitis.

Figure 5. Forest Plot for PD outcome in peri-implantitis.

Figure 6. Forest Plot for BOP outcome in peri-implantitis.

Figure 7. Forest Plot for PD outcome in both peri-implant mucositis and peri-implantitis.

Figure 8. Forest Plot for BOP outcome in both peri-implant mucositis and peri-implantitis.

Figure 9. Review of authors' judgements about each risk of bias item presented as a a) percentage in a graph and b) summary for each included study.

Appendix table 1. List of excluded articles.

Appendix Figure 1. PRISMA flowchart of the screening process.

Appendix I. Search protocol used in the systematic review.

Appendix II. Extracted information on study characteristics. 


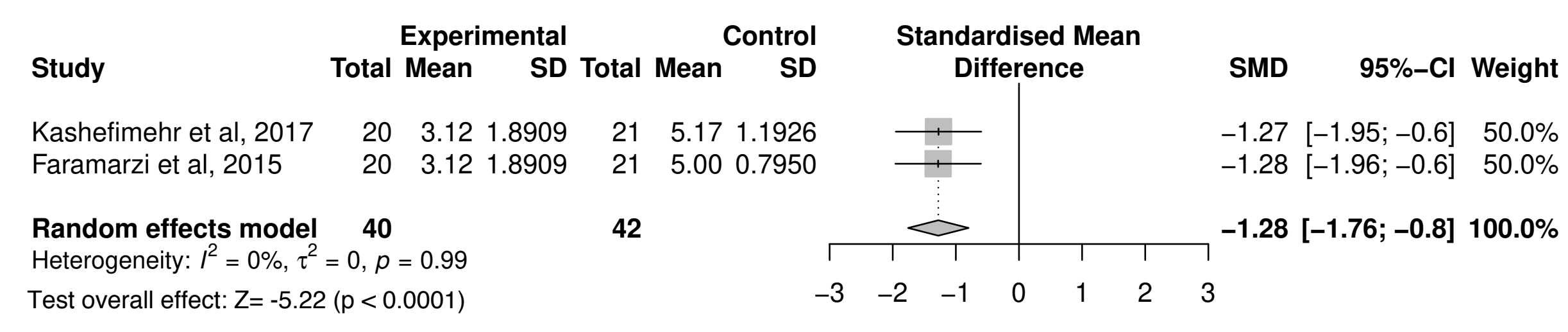




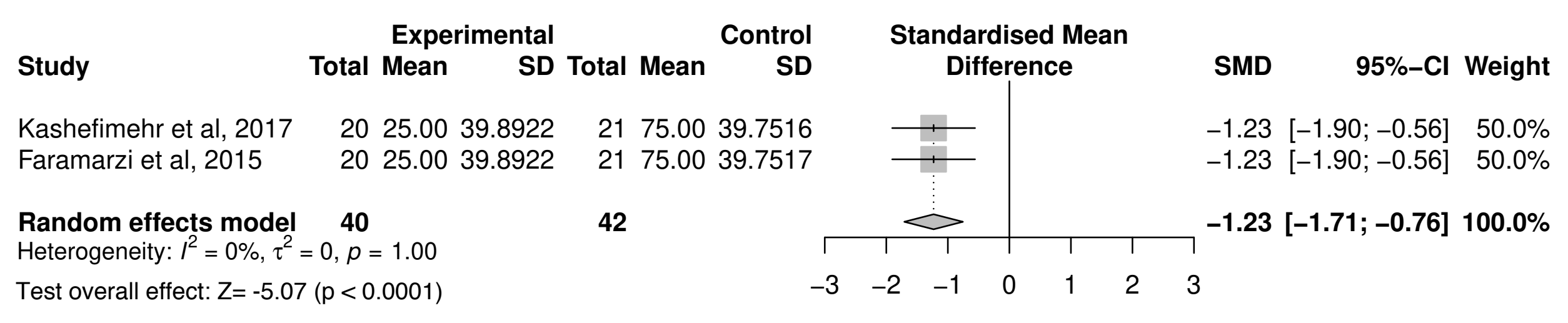




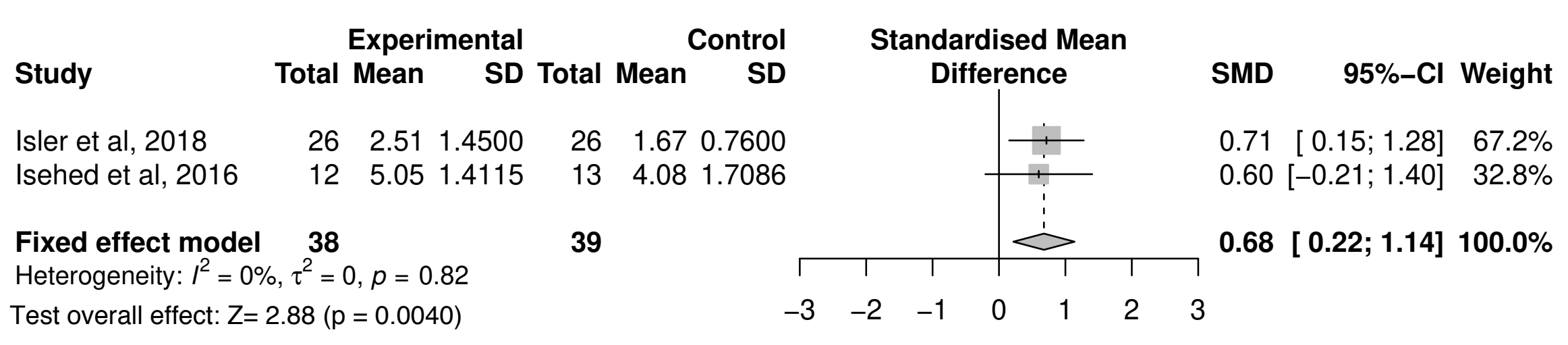




\section{Study}

Isler et al, 2018

Hamzacebi et Hamzacebi et al,
Isehed et al, 2016

Random effects model 57

Heterogeneity: $I^{2}=89 \%, \tau^{2}=0.9510, p<0.0$

Test overall effect: $Z=0.14(p=0.887)$
Experimental Control Standardised Mean

Total Mean SD Total Mean SD

$\begin{array}{llllll}26 & 3.71 & 1.0900 & 26 & 2.70 & 0.8000\end{array}$

$\begin{array}{llllll}26 & 3.71 & 1.0900 & 26 & 2.70 & 0.8000 \\ 19 & 3.30 & 0.4900 & 19 & 3.71 & 0.4200\end{array}$

$\begin{array}{rrrrrr}19 & 3.30 & 0.4900 & 19 & 3.71 & 0.4200 \\ 12 & 4.31 & 1.6109 & 13 & 4.19 & 2.0234\end{array}$

58

234$$
\text { Difference }
$$

SMD

95\%-Cl Weight

$1.04 \quad[0.46 ; 1.62] \quad 34.4 \%$

$-0.88[-1.55 ;-0.21] \quad 33.5 \%$

$0.07[-0.72 ; 0.85] 32.2 \%$

$0.08[-1.09 ; 1.26] 100.0 \%$ 


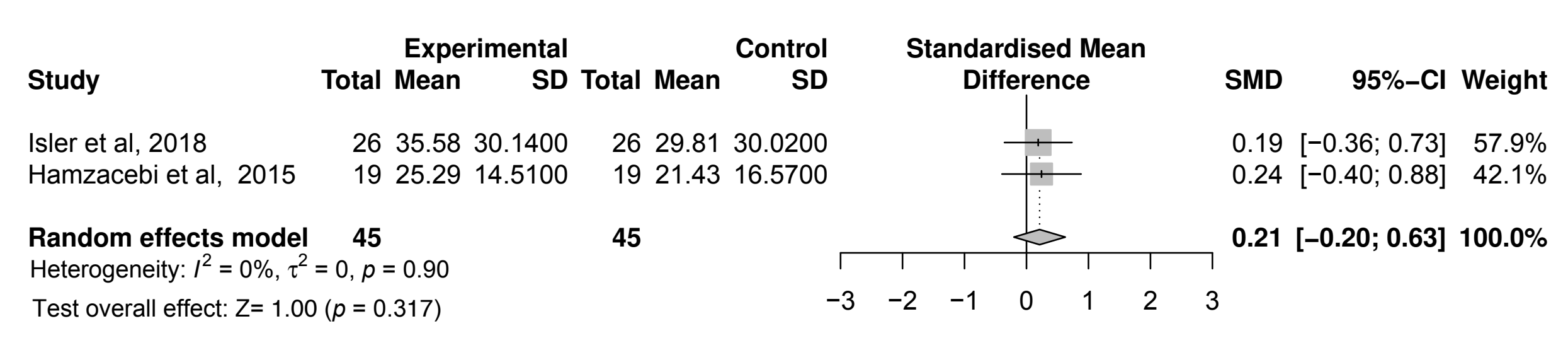




\begin{tabular}{|c|c|c|c|c|c|c|c|c|c|c|}
\hline \multirow[b]{2}{*}{ Study } & \multicolumn{2}{|c|}{ Experimental } & & \multicolumn{2}{|r|}{ Control } & \multirow{2}{*}{\multicolumn{2}{|c|}{$\begin{array}{l}\text { Standardised Mean } \\
\text { Difference }\end{array}$}} & \multirow[b]{2}{*}{ SMD } & \multirow[b]{2}{*}{$95 \%-C$} & \multirow[b]{2}{*}{ Weigh } \\
\hline & Total & Mean SD & Total & Mean & SD & & & & & \\
\hline Isler et al, 2018 & 26 & 3.711 .0900 & 26 & 2.70 & 0.8000 & & 1 & 1.04 & [ $0.46 ; 1.62]$ & $20.6 \%$ \\
\hline Hamzacebi et al, 2015 & 19 & 3.300 .4900 & 19 & 3.71 & 0.4200 & + & & -0.88 & {$[-1.55 ;-0.21]$} & $20.1 \%$ \\
\hline Isehed et al, 2016 & 12 & $4.31 \quad 1.6109$ & 13 & 4.19 & 2.0234 & & & 0.07 & {$[-0.72 ; 0.85]$} & $19.3 \%$ \\
\hline Kashefimehr et al, 2017 & 20 & 3.121 .8909 & 21 & 5.17 & 1.1926 & 1 & & -1.27 & {$[-1.95 ;-0.60]$} & $20.0 \%$ \\
\hline Faramarzi et al, 2015 & 20 & 3.121 .8909 & 21 & 5.00 & 0.7950 & 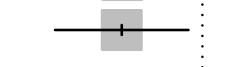 & & -1.28 & {$[-1.96 ;-0.60]$} & $20.0 \%$ \\
\hline $\begin{array}{l}\text { Random effects model } \\
\text { Heterogeneity: } I^{2}=90 \%, \tau\end{array}$ & $\begin{aligned} & 97 \\
= & 1.0\end{aligned}$ & $511, p<0.01$ & 100 & & & $\widetilde{ }$ & $\frac{1}{1}$ & $7^{-0.46}$ & {$[-1.41 ; 0.49]$} & $100.0 \%$ \\
\hline Test overall effect: $Z=-0.9$ & $5(p=0$ & & & & -3 & $-2-1$ & 1 & 3 & & \\
\hline
\end{tabular}




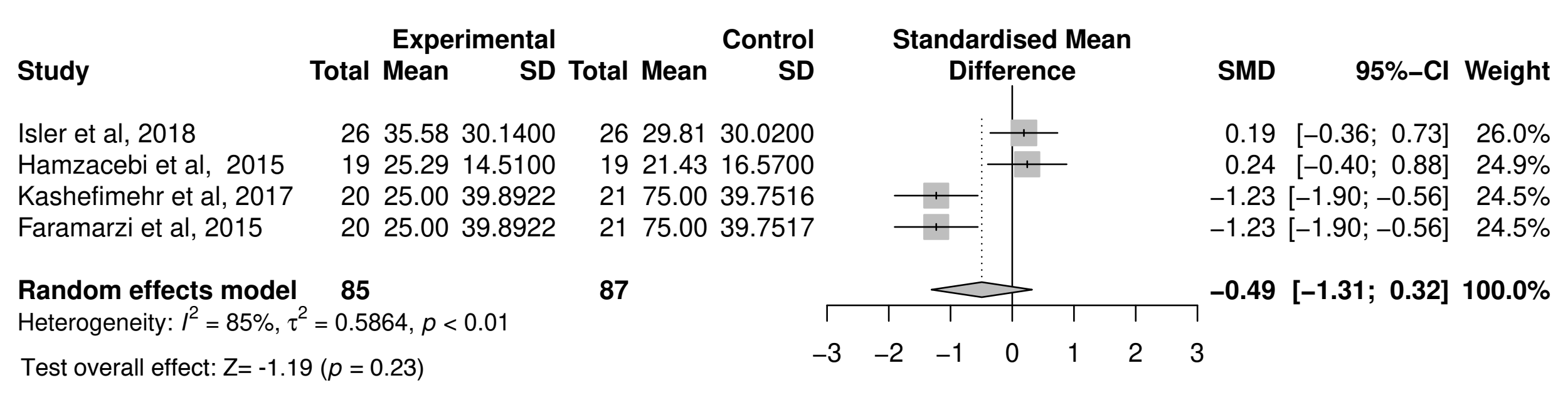

